\title{
Standing and propagating waves in cubically nonlinear media
}

\author{
Bengt O. Enflo*, Claes M. Hedberg ${ }^{\dagger}$ and Oleg V. Rudenko ${ }^{\dagger}$ \\ ${ }^{*}$ Department of Mechanics, Kungl. Tekniska Högskolan, \\ S-10044 Stockholm, Sweden \\ e-mail: benflo@mech.kth.se \\ phone: Int+46 8 7907156, fax: Int+468 7969850 \\ ${ }^{\dagger}$ Mechanical Engineering Department, Blekinge Institute of \\ Technology, S-371 79 Karlskrona, Sweden
}

\begin{abstract}
The paper has three parts. In the first part a cubically nonlinear equation is derived for a transverse finite-amplitude wave in an isotropic solid. The cubic nonlinearity is expressed in terms of elastic constants. In the second part a simplified approach for a resonator filled by a cubically nonlinear medium results in functional equations. The frequency response shows the dependence of the amplitude of the resonance on the difference between one of the resonator's eigenfrequencies and the driving frequency. The frequency response curves are plotted for different values of the dissipation and differ very much for quadratic and cubic nonlinearities. In the third part a propagating $\mathrm{N}$-wave is studied, which fulfils a modified Burgers' equation with a cubic nonlinearity. Approximate solutions to this equation are found for new parts of the wave profile.
\end{abstract}

Keywords: Cubic nonlinear media, nonlinear acoustic resonator, cubic resonator, $\mathrm{N}$-wave propagation

PACS: 43.25.Gf, 43.25.Dc

\section{INTRODUCTION}

Nonlinear acoustical waves in dissipative media have been extensively studied and model equations describing weakly nonlinear, weakly dissipative waves have been solved $[1,2,3]$. In most cases the nonlinearity is quadratic and the model equations are Burgers' or generalized Burgers' equations [4]. An interesting problem of principial interest is to investigate how the methods used for quadratic nonlinearities can be applied to cubic nonlinearities. Results of such investigations show new features in comparison with quadratic nonlinearities.

The quadratic nonlinearity is characteristic for waves in fluids [3] and also for longitudinal waves in solids [5]. However, transverse wave propagation is modelled by a cubically nonlinear wave equation, since the quadratic nonlinearity cancels [6]. The derivation of this equation is the contents of section 2 . Two examples of cubically nonlinear wave theory are studied. In section 3 standing waves in a cubically nonlinear resonator are studied and the frequency response function is calculated and compared with the corresponding function in the quadratically nonlinear case [7]. In section 4 cubically nonlinear propagation of $\mathrm{N}$-waves is studied using methods developed for quadratically nonlinear waves [8]. 


\section{PHYSICAL BACKGROUND}

The equation of motion of a continuous medium is [5]

$$
\rho_{0} \frac{\partial^{2} U_{i}(\vec{a}, t)}{\partial t^{2}}=\frac{\partial}{\partial a_{j}}\left(P_{i j}+D_{i j}\right)
$$

where

$\rho_{0}$ is the density in the undeformed state,

$U_{i}$ is the displacement, $U_{i}=x_{i}-a_{i}$, of the particle originally at $a_{i}$ (Lagrangian coord.), $P_{i j}$ is the stress tensor,

$D_{i j}$ is the dissipative stress tensor.

The elastic energy $W$ is expanded in the invariants of the strain tensor $E_{i j}$,

$$
E_{i j}=\frac{1}{2}\left(\frac{\partial U_{i}}{\partial a_{j}}+\frac{\partial U_{j}}{\partial a_{i}}+\frac{\partial U_{k}}{\partial a_{i}} \frac{\partial U_{k}}{\partial a_{j}}\right),
$$

to the fourth order [6]:

$$
\begin{gathered}
\rho_{0} W=\left(\frac{K}{2}-\frac{\mu}{3}\right)\left(E_{k k}\right)^{2}+\mu E_{i j} E_{j i}+\frac{C}{3}\left(E_{k k}\right)^{3}+B E_{k k} E_{i j} E_{j i}+\frac{A}{3} E_{i j} E_{j k} E_{k i} \\
+H\left(E_{k k}\right)^{4}+G\left(E_{i j} E_{j i}\right)^{2}+F\left(E_{k k}\right)^{2} E_{i j} E_{j i}+E E_{k k} E_{i j} E_{j l} E_{l i}+D E_{i j} E_{j k} E_{k l} E_{l i} .
\end{gathered}
$$

Using the relation

$$
P_{i j}=\rho_{0} \frac{\partial W}{\partial\left(\frac{\partial U_{i}}{\partial a_{j}}\right)}
$$

we obtain from (1) and (3) the equations of motion, with the notation $U_{i j}=\frac{\partial U_{i}}{\partial a_{j}}$,

$$
\begin{array}{r}
\rho_{0}\left\{\frac{\partial^{2} U_{i}}{\partial t^{2}}-c_{t}^{2} \frac{\partial^{2} U_{i}}{\partial a_{k}^{2}}-\left(c_{l}^{2}-c_{t}^{2}\right) \frac{\partial U_{k k}}{\partial a_{i}}\right\} \\
=\frac{\partial D_{i}}{\partial t}+\frac{\partial}{\partial a_{s}}\left\{\left(\frac{K}{2}-\frac{\mu}{3}\right)\left(U_{k l} U_{k l} \delta_{i s}+2 U_{k k} U_{i s}+U_{k l} U_{k l} U_{i s}\right)\right. \\
\left.+\mu\left(U_{i k} U_{s k}+U_{i k} U_{k s}+U_{k i} U_{k s}+U_{i k} U_{r k} U_{r s}\right)+\ldots\right\},
\end{array}
$$

where "..." stands for terms of second and third order in $U_{i k}$ with coeffi cients $A, B, C, D$, $E, F, G, H$, and $c_{t}$ and $c_{l}$ are the transverse and longitudinal wave propagation velocities respectively,

$$
c_{t}^{2}=\frac{\mu}{\rho_{0}}, c_{l}^{2}=\frac{K+\frac{4 \mu}{3}}{\rho_{0}} .
$$

The dissipative term $\frac{\partial D_{i}}{\partial t}$ in (5) is obtained from

$$
D_{i}=\eta\left(\frac{\partial^{2} U_{i}}{\partial a_{k}^{2}}+\frac{\partial^{2} U_{k}}{\partial a_{i} \partial a_{k}}\right)+\left(\zeta-\frac{2}{3} \eta\right) \frac{\partial^{2} U_{k}}{\partial a_{i} \partial a_{k}},
$$


where $\eta, \zeta$ are the shear and bulk viscosities respectively.

With the scalings

$$
\begin{array}{r}
\bar{a}_{1}=\varepsilon^{\frac{1}{2}} a_{1}, \quad \bar{a}_{2}=\varepsilon^{\frac{1}{2}} a_{2}, \quad \bar{a}_{3}=\varepsilon a_{3} \\
\bar{U}_{1}=\varepsilon^{-\frac{1}{2}} U_{1}, \quad \bar{U}_{2}=\varepsilon^{-\frac{1}{2}} U_{2}, \quad \bar{U}_{3}=\varepsilon^{-1} U_{3} \\
\bar{\eta}=\varepsilon^{-1} \eta, \quad \bar{\zeta}=\varepsilon^{-1} \zeta
\end{array}
$$

a nonlinear beam equation can be derived [6] for transverse waves, vibrating in the 1-direction and propagating in the 3-direction. In this case the quadratic nonlinearity cancels and the equation for $\frac{\partial \bar{U}_{1}}{\partial t}=V$ becomes

$$
\frac{\partial}{\partial \tau}\left\{\frac{\partial V}{\partial z}-\frac{\beta}{c_{t}^{3}} V^{2} \frac{\partial V}{\partial \tau}-\delta \frac{\partial^{2} V}{\partial \tau^{2}}\right\}=\frac{c_{t}}{2}\left\{\frac{\partial^{2}}{\partial x^{2}}+\frac{\partial^{2}}{\partial y^{2}}\right\} V,
$$

where $(x, y, z, \tau)$ is given as $\left(\bar{a}_{1}, \bar{a}_{2}, \bar{a}_{3}, t-\frac{\bar{a}_{3}}{c_{3}}\right)$ and $\delta$ and $\beta$ are given as

$$
\begin{gathered}
\delta=\frac{\bar{\eta}}{2 \rho_{0} c_{t}^{3}} \\
\beta=\frac{3}{2 \rho_{0} c_{t}^{2}}\left\{\frac{K}{2}+\frac{2 \mu}{3}+B+\frac{A}{2}+G+\frac{D}{2}-\frac{\left(\frac{K}{2}+\frac{2 \mu}{3}+\frac{3 B}{4}+\frac{A}{4}\right)\left(K+\frac{4 \mu}{3}+B+\frac{A}{2}\right)}{K+\frac{\mu}{3}}\right\} .
\end{gathered}
$$

The quotient term in (11), because of which $\beta$ can be negative, is missing in the paper by Zabolotskaya [6]. The equation (9) is studied by Rudenko and Sapoznikov [9]. If $K$ and $\mu$ are the only elastic constants different from zero in (11) we obtain using (6):

$$
\beta=-\frac{3}{4} \frac{c_{l}^{2}}{c_{l}^{2}-c_{t}^{2}}
$$

Thus $\beta$ is negative for the simplest solid materials.

\section{STANDING WAVES IN A CUBICALLY NONLINEAR RESONATOR}

Neglecting dissipation and transverse extension Eq. (9) becomes

$$
\frac{\partial V}{\partial z}-\frac{\beta}{c^{3}} V^{2} \frac{\partial V}{\partial \tau}=0
$$

where $c_{t}$ is replaced by $c$ in order that Eq. (13) be applied to other physical phenomena than transverse waves in solids. The choice of the negative sign in the defi nition of $\tau$ means that Eq. (13) describes rightgoing waves. Standing waves in a resonator are 
composed by waves propagating in both directions. A wave equation, which gives Eq. (13) when specialized to rightgoing waves, is (change $z$ into $x$ ):

$$
\frac{\partial^{2} V}{\partial x^{2}}-\frac{1}{c^{2}} \frac{\partial^{2} V}{\partial t^{2}}=-\frac{2 \beta}{3 c^{4}} \frac{\partial^{2} V^{3}}{\partial t^{2}} .
$$

The equation with a quadratic nonlinearity corresponding to the cubic nonlinear equation (14) is studied for a resonator by the present authors [7]. The resonator boundary conditions are

$$
V(x=0, t)=A \sin \omega t, \quad V(x=L, t)=0 .
$$

In analogy with the solution attempted by the present authors for a quadratic nonlinearity [7] we attempt a solution to Eq. (14) in the form

$$
\begin{array}{r}
V=V_{+}+V_{-}, V_{ \pm}= \pm F\left(\omega t \mp \frac{\omega}{c}(x-L) \pm \frac{\beta \omega}{c^{3}}\left(I+F^{2}\right)(x-L)\right), \\
I=\left\langle V_{ \pm}^{2}\right\rangle .
\end{array}
$$

Inserting (16) into the boundary conditions (15) gives a functional equation

$$
\begin{aligned}
F\left(\omega t+k L-\frac{\beta \omega}{c^{3}}\left(I+F^{2}\right)(x-L)\right)-F(\omega t-k L & \left.+\frac{\beta \omega}{c^{3}}\left(I+F^{2}\right)(x-L)\right) \\
& =A \sin \omega t, \quad k=\frac{\omega}{c} .
\end{aligned}
$$

The functional equation (17) can be reduced to a differential equation with dimensionless variables:

$$
\frac{\partial W}{\partial t}+\left(\Delta-\pi \beta J-\pi \beta W^{2}\right) \frac{\partial W}{\partial \xi}-D \frac{\partial^{2} W}{\partial \xi^{2}}=\frac{M}{2} \sin \xi
$$

with

$$
W=\frac{F}{c}, M=\frac{A}{c}, \xi=\omega t+\pi, T=\frac{\omega t}{\pi}, \quad J=\frac{I}{c^{2}} .
$$

The discrepancy $\Delta$ in Eq. (18) is defi ned as

$$
\Delta=\frac{\left(\omega-\omega_{0}\right)}{\omega_{0}}
$$

with $\omega=\frac{\pi L}{c}$ (lowest resonance frequency of the resonator).

The dissipation coeffi cient $D$ in Eq. (18) is defi ned as

$$
D=\frac{b \omega L}{2 c^{3} \rho_{0}}<<1,
$$

where the absorption coeffi cient $b(\sim \eta$ and $\zeta)$ can be introduced in Eq. (18) in analogy with its occurrence in Burgers' equation [1]. 


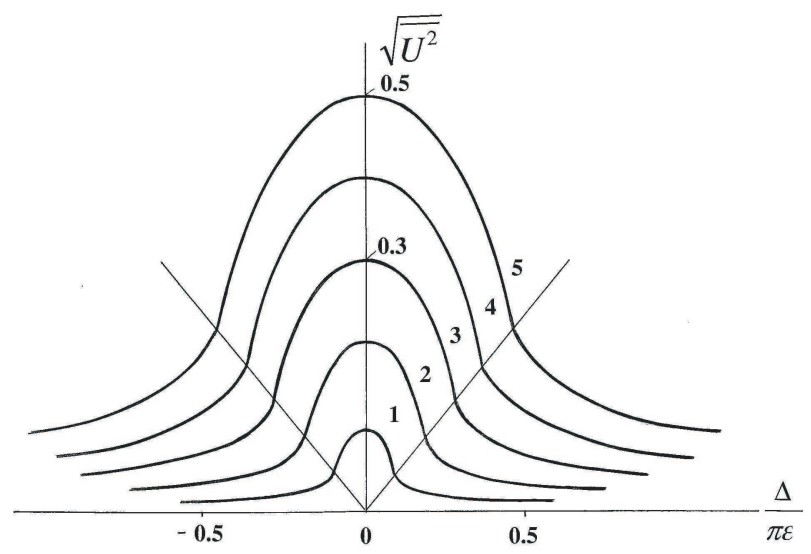

FIGURE 1. Quadratic resonator root-mean-square velocity as function of discrepancy parameter $\Delta /(\pi \varepsilon)$. The curves are for different boundary vibration amplitudes.

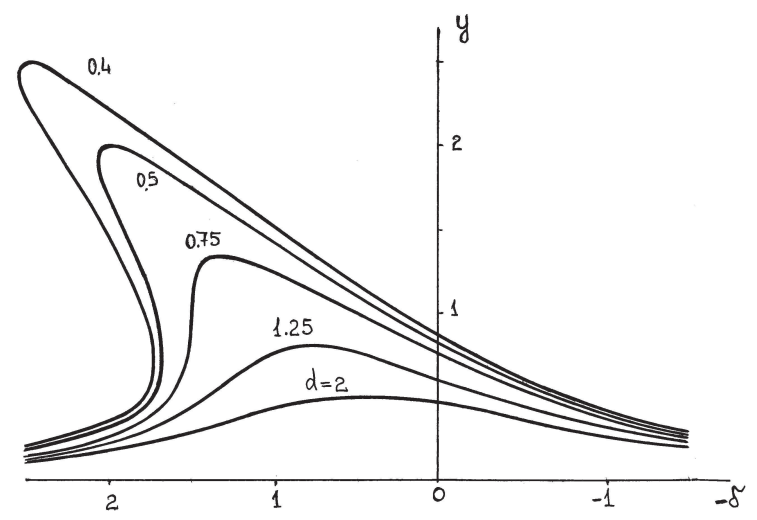

FIGURE 2. The cubic resonator frequency response as a function of discrepancy parameter $\delta$. The curves are (from above) for different absorptions: $\mathrm{d}=0.4,0.5,0.75,1.25,2$.

Frequency response functions for a quadratic and a cubic nonlinear resonator are plotted in Fig. 1 and Fig. 2 respectively. In Fig. 1 we plot the rms normalized particle velocity $\sqrt{\overline{W^{2}}}$ as function of the normalized discrepancy $\frac{\Delta}{\pi \varepsilon}$, where $\varepsilon$ is the quadratic nonlinearity parameter. Curves 1-5 are constructed for different values of boundary vibration $\left[\frac{M}{\pi \varepsilon} \cdot 10^{2}=1,4,9,16\right.$ and 25]. In Fig. 2 a frequency response function $y(\delta)$ with

$$
y=\frac{I}{c^{2}}\left(\frac{3 \sqrt{2} \pi \beta}{M}\right)^{\frac{2}{3}}, \quad \delta=\Delta\left(\frac{16}{3 \pi \beta M^{2}}\right)^{\frac{1}{3}}
$$

is constructed for different values of the normalized absorption coeffi cient $\left[d=D\left(\frac{3 \sqrt{2} \pi \beta}{M}\right)^{\frac{1}{3}}=2,1.25,0.75,0.5\right.$ and 0.4$]$. 


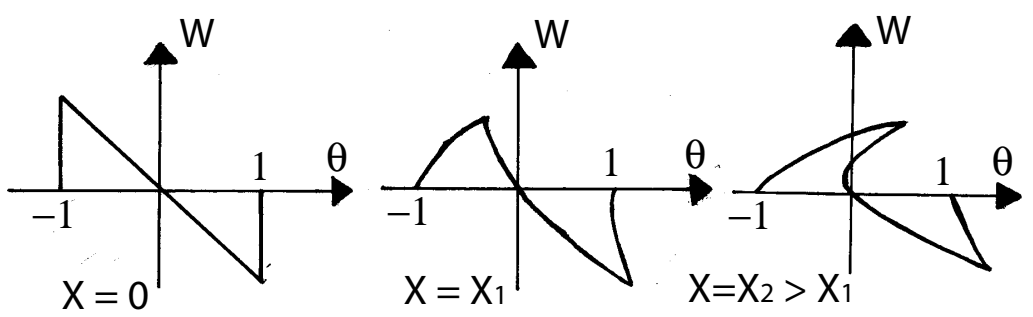

FIGURE 3. Schematic evolution of an original N-wave in a cubically nonlinear medium.

\section{PROPAGATING N-WAVES IN A CUBICALLY NONLINEAR MEDIUM}

The modifi ed Burgers' equation for plane waves

$$
\frac{\partial V}{\partial z}-\frac{\beta}{c_{t}^{3}} V^{2} \frac{\partial V}{\partial \tau}=\delta \frac{\partial^{2} V}{\partial \tau^{2}}
$$

following from (9), has to be made dimensionless. To this end we introduce a fundamental period $v=t_{0}^{-1}$, where $t_{0}$ is the duration of the $\mathrm{N}$-wave at the boundary, and the velocity amplitude $V_{0}$ at the boundary. The dimensionless equation derived from (23) is

$$
\frac{\partial W}{\partial X}+W^{2} \frac{\partial W}{\partial \theta}=\varepsilon \frac{\partial^{2} W}{\partial \theta^{2}}
$$

with

$$
W=\frac{V}{V_{0}}, \quad X=\frac{2|\beta| V_{0}^{2} v}{c_{t}^{3}} z, \quad \theta=2 \nu \tau, \varepsilon=\frac{\bar{\eta} v}{|\beta| \rho_{0} V_{0}^{2}} .
$$

We assume $\beta<0$ and $\varepsilon<<1$.

The equation (24) has been studied by Lee-Bapty and Crighton [10]. The qualitative deformation of an original $\mathrm{N}$-wave for increasing $X$-values according to Eq. (24) with $\varepsilon \rightarrow 0$ is shown in Fig. 3 .

The multivalued parts of the profi le in the last picture of Fig. 3 are replaced [10] by a tail shock at $\theta=C_{t}(X)=-1+d^{2} X^{\frac{1}{4}}$ and a head shock at $\theta=C_{h}(X)=-1+3 d^{2} X^{\frac{1}{4}}$ with $d \approx 0.95$. The structures of the shocks and of the tail at $\theta \approx-1$ are found by rescaling Eq. (24), so that the righthand side is no longer small. By this procedure seven parts of the profi le are discerned, satifying different scaled versions of Eq. (24) with different dominant terms (Fig. 4).

The new results in the present paper concern part 1 (left tail) and part 2 (connection at $\theta \approx-1$ between left tail and left curve). For parts 3 (left curve), 4 (tail shock), 5 (right curve) and 6 (head shock) analytic lowest approximation solutions are given by Lee-Bapty and Crighton [10]. For part 7 (right tail) an analytic solution is still not yet found. 


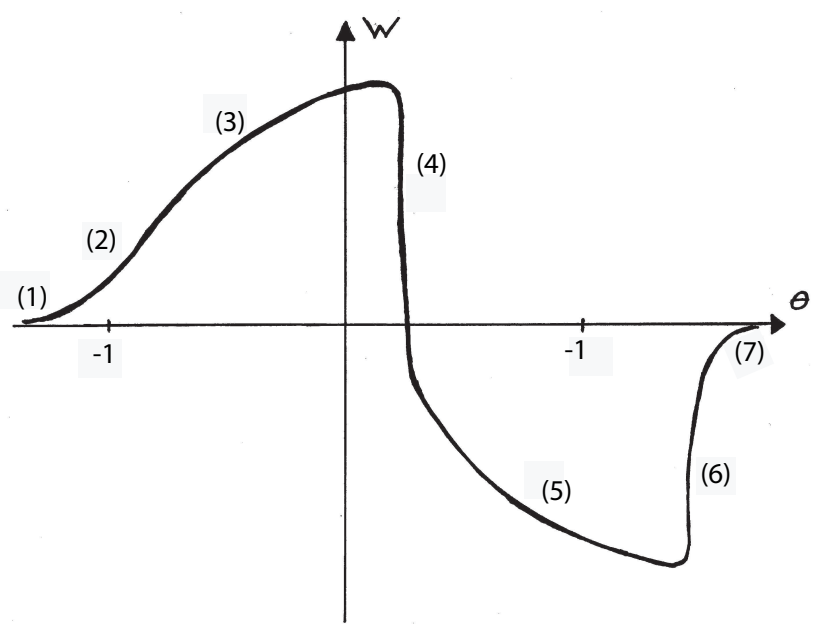

FIGURE 4. The seven parts of the deformed N-wave in a cubically nonlinear medium.

We fi rst attempt to fi nd a solution for part 2, because this solution has to be consistent with the part 1 and part 3 solutions. The scalings

$$
X^{*}=\varepsilon^{\frac{1}{2}} X, \quad \theta^{*}=\frac{\theta+1}{\varepsilon^{\frac{1}{2}}}, \quad W^{*}=\varepsilon^{\frac{1}{2}} W=W_{0}^{*}+O\left(\varepsilon^{\frac{1}{2}}\right)
$$

are inserted into (24) and give in lowest order

$$
W_{0}^{* 2} \frac{\partial W_{0}^{*}}{\partial \theta^{*}}=\frac{\partial^{2} W_{0}^{*}}{\partial \theta^{* 2}}
$$

The solution of (27) with the necessary consistency properties is

$$
W_{0}=\varepsilon^{-\frac{1}{2}} W_{0}^{*}=\sqrt{\frac{3}{2}} \frac{\varepsilon^{\frac{1}{4}}}{\sqrt{C-\varepsilon^{\frac{1}{2}}(\theta+1)}},
$$

where the constant $C$ can be determined numerically. The solution (28) is valid for

$$
\begin{gathered}
|\theta+1|<<2 \varepsilon^{\frac{1}{2}} X^{\frac{1}{2}}, \quad \theta<-1 \\
\theta+1<\frac{3}{2} \varepsilon^{\frac{1}{2}} \frac{X}{C}, \quad \theta>-1 .
\end{gathered}
$$

The independence of the solution (28) on $X$ is seen in the numerically calculated solution in Fig. 5, where all profiles for different $X$ have approximately the same value for $\theta=-1$.

For part 1 (left tail) we make the scalings

$$
\xi=\varepsilon^{\frac{3}{2}} X, \quad \zeta=-\varepsilon^{\frac{1}{4}}(\theta+1), \quad U=\varepsilon^{-\frac{7}{8}} W=U_{0}+O\left(\varepsilon^{\frac{1}{2}}\right),
$$




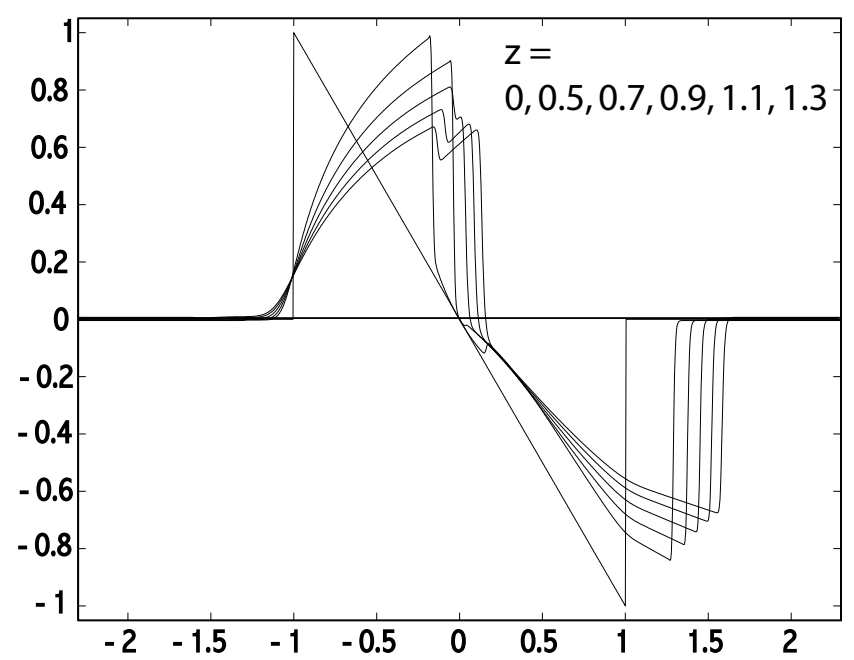

FIGURE 5. Numerical solutions of Eq. (24), evaluated with $\varepsilon=0.005$ for $X=0,0.5,0.7,0.9,1.1,1.3$.

which are inserted into (24) and give a lowest order linear equation

$$
\frac{\partial U_{0}}{\partial \xi}=\frac{\partial^{2} U_{0}}{\partial \zeta^{2}}
$$

The equation (32) is identically solved by the integral representation

$$
\begin{array}{r}
U_{0}=K \int_{0}^{\infty} h(\lambda) \exp \left(\mathrm{i} \lambda \zeta-\lambda^{2} \xi\right) d \lambda+\text { c.c. } \\
K=\sqrt{\frac{3}{8 \pi}} \varepsilon^{-\frac{1}{2}}, h(\lambda)=\lambda^{-\frac{1}{2}}
\end{array}
$$

where c.c. stands for complex conjugated and $K$ and $h(\lambda)$ are chosen so that the solution (33) matches the solution (28). This is seen by evaluating the integral in (33) by the steepest descent method with $X$ and $\theta$ inserted from (31) with the result

$$
W_{0}=\varepsilon^{\frac{7}{8}} U_{0}=\sqrt{\frac{3}{2}}|\theta+1|^{-\frac{1}{2}} \exp \left(-\frac{(\theta+1)^{2}}{4 \varepsilon X}\right) .
$$

Solutions (28) and (34) match each other in the region where (29) is fulfilled together with

$$
|\theta+1|>>C \varepsilon^{-\frac{1}{2}}
$$

For part 3 (left curve) the expansion $W=W_{0}+O(\varepsilon)$ gives using (24) [10]

$$
\frac{\partial W_{0}}{\partial X}+W_{0}^{2} \frac{\partial W_{0}}{\partial \theta}=0 \Longrightarrow W_{0}=\sqrt{\frac{\theta+1}{X}} .
$$

For the solution (36) to be valid we must require (cf. Eq. 24)

$$
\varepsilon\left|\frac{\partial^{2} W_{0}}{\partial \theta^{2}}\right|<<\left|\frac{\partial W_{0}}{\partial X}\right| \Longrightarrow \frac{(\theta+1)^{2}}{\varepsilon X}>>1 .
$$


Thus for $X=O(1)$ the transition from the solution (37) to the solution (28) is completed at $\theta+1=O\left(\varepsilon^{\frac{1}{2}}\right)$. A representation similar to (34) for the right tail remains to be found, as well as an exponentially decreasing profi le at both ends for $X>>\varepsilon^{-2}$ (cf. the scaling (31)).

\section{REFERENCES}

1. O.V. Rudenko, and S.I. Soluyan, Theoretical Foundations of Nonlinear Acoustics, Plenum, Consultants Bureau, New York, 1977.

2. P.L. Sachdev, Nonlinear Diffusive Waves, Cambridge University Press, Cambridge, 1987.

3. B.O. Enflo, and C.M. Hedberg, Theory of Nonlinear Acoustics in Fluids, Kluwer, Dordrecht, 2002.

4. D.G. Crighton, and J.F. Scott, Asymptotic solutions of model equations in nonlinear acoustics. Phil. Trans. R. Soc. Lond. A 292, 101-134 (1979).

5. A.N. Norris, "Finite amplitude waves in solids", in Nonlinear Acoustics, edited by M.F. Hamilton, and D.T. Blackstock, Academic Press, San Diego, 1998, pp. 263-277.

6. E.A. Zabolotskaya, Sound beams in a nonlinear isotropic solid, Sov. Phys. Acoust. 32, 296-299 (1986).

7. B.O. Enflo, C.M. Hedberg, and O.V. Rudenko, Resonant properties of a nonlinear dissipative layer excited by a vibrating boundary: Q-factor and frequency response, J. Acoust. Soc. Am. 117, 601-612 (2005).

8. B.O. Enflo, On the connection between the asymptotic waveform and the fading tail of an initial N-wave in nonlinear acoustics, Acustica - Acta Acustica 84, 401-413 (1998).

9. O.V. Rudenko, and O.A. Sapoznikov, Wave beams in cubically nonlinear nondispersive media, JETP 79, 395-413 (1994).

10. I.P. Lee-Bapty, and D.G. Crighton, Nonlinear wave motion governed by the modified Burgers equation. Phil. Trans. R. Soc. Lond. A 323, 173-209 (1987). 